\title{
Outcome of Prolonged Cardiac Arrest Under Extracorporeal Cardiopulmonary Resuscitation Due to Acute Myocardial Infarction: Complete vs Culprit Revascularization
}

\author{
Hsi-Yu Yu \\ National Taiwan University Hospital \\ Chih-Hsien Wang \\ National Taiwan University Hospital \\ Lian-Yu Lin \\ National Taiwan University Hospital \\ Jou-Wei Lin \\ National Taiwan University Hospital Yun Lin Branch \\ Nai-Hsin Chi \\ National Taiwan University Hospital \\ Yi-Chih Wang \\ National Taiwan University Hospital \\ Heng-Wen Chou \\ National Taiwan University Hospital \\ Nai-Kuan Chou \\ National Taiwan University Hospital \\ Juey-Jen Hwang Hwang \\ National Taiwan University Hospital \\ YIH SHARNG CHEN ( $\nabla$ yschen1234@gmail.com ) \\ National Taiwan University Hospital https://orcid.org/0000-0003-3846-8162
}

Original research

Keywords: Extracorporeal membrane oxygenation, cardiopulmonary resuscitation, extracorporeal cardiopulmonary resuscitation, acute myocardial infarction, percutaneous coronary intervention, coronary artery bypass grafting

Posted Date: July 27th, 2020

DOI: https://doi.org/10.21203/rs.3.rs-47380/v1 
License: (c) (i) This work is licensed under a Creative Commons Attribution 4.0 International License. Read Full License 


\section{Abstract \\ Background:}

Immediate revascularization of infarct-related vessel (culprit strategy) is effective in patients with acute myocardial infarction (AMI) with cardiogenic shock. However, for AMI patients complicated with prolonged cardiac arrest under extracorporeal membrane oxygenation resuscitation (ECPR), whether culprit revascularization (IR) or complete revascularization (CR) is associated with better clinical outcome is not known.

\section{Methods:}

Patients with AMI complicated with prolonged cardiac arrest under ECMO support between 2006 and 2016 were included and were grouped by the status of revascularization completeness in three coronary territories into IR and CR groups. The primary endpoint is favorable neurological outcomes at hospital discharge. The second endpoint is the probability of a composite of major adverse cardiac and cerebral events (MACCE) at 1-year follow-up.

\section{Results:}

A total of 90 patients (32 IR and $58 \mathrm{CR}$ ) were included. Favorable neurological outcomes at hospital discharge were $21.9 \%$ and $37.9 \%$ in IR and $C R$ patients, respectively $(P=0.121$.) Multivariate logistic regression analysis did not reveal $\mathrm{CR}$ a significant risk factor (odds ratio: 1.82, 95\% confidence interval [CI]: $0.70-4.77, P=0.221$ ). One-year freedom from MACCE probabilities were $24.6 \%$ and $42.4 \%$ in IR and $C R$ patients, respectively $(P=0.051$. $)$ Cox regression analysis revealed that $C R$, in addition to age, low-flow duration, and initial shockable rhythm, was a risk factor (hazard ratio: $0.53,95 \% \mathrm{Cl}: 0.31-0.91, \mathrm{P}=0.020$ ).

\section{Conclusion:}

For patients with AMI under ECPR, culprit strategy had similar result to that of CR strategy in one-year outcome.

\section{Introduction}

Acute myocardial infarct (AMI) complicated with cardiogenic shock and prolonged cardiopulmonary resuscitation (CPR) needing extracorporeal membrane oxygenation (ECMO) support is one of the most detrimental conditions for patients and the most challenging situations for medical personnel. Because rapid death due to persistent shock is likely in this condition, several clinical trials, such as CHEER trial(1) and Prague Out-of-Hospital Cardiac Arrest (OHCA) study,(2) have advocated immediate revascularization of occluded coronary arteries. However, these studies have not compared the probabilities of hospital 
survival and favorable neurological outcomes associated with partial revascularization (PR) or complete revascularization (CR). Theoretically, CR of all stenotic coronary arteries, either through percutaneous coronary intervention $(\mathrm{PCl})$ or coronary artery bypass grafting (CABG), may provide the highest chance of heart function recovery, but it may also induce end-organ damage because of the prolonged procedural time and repeated hypoperfusion of the brain and other vital organs during revascularization. The CULPRIT-SHOCK study demonstrated a similar survival rates between PR and CR patients but with a higher incidence of repeated coronary intervention in PR patients during 1-year follow-up.

In the present study, the effects of revascularization strategy on hospital survival and 1-year outcomes for an extremely critical AMI patients, namely patients with cardiac arrest and requiring ECPR, were evaluated. The results of the study can contribute to decision-making protocols(3) for the detrimental AMI complicated by profound cardiogenic shock.

\section{Methods}

All adult patients (age $\geq 18$ years) who had received ECPR and emergency coronary angiography at our institution between 2006 and 2016 were included. All demographic and clinical data were prospectively collected and systemically reviewed under institutional approval (NTUH-9561707007.) The present study was approved by the review board of our institute (NTUH 201810079 RIN).

The contraindications of ECPR, modified from previous reports, $(4,5)$ are listed in supplement Table 1 . The decision to perform coronary angiography after ECPR was made by the consulted cardiologist based on a combination of multiple factors, including presenting symptoms before cardiac arrest, risk factors for coronary artery disease (CAD), and initial and serial electrocardiography (EKG) findings suspecting AMI or a VT or VF rhythm. Emergency coronary angiography was performed on emergency status after exclusion of acute aortic dissection or massive intracranial hemorrhage detected by computed tomography scanning. After confirming the diagnosis of CAD, the decision of emergency PCl or CABG surgery was determined by a discussion between the on-duty cardiologist and cardiac surgeon ( 10 cardiologists and 5 cardiac surgeons on call $24 \mathrm{~h}$ a day and 7 days a week) in which multiple factors, including anatomy of the coronary arteries, culprit coronary lesion pathology, general conditions before the index ECPR event, low-flow duration before ECMO, and the patients' and relatives' will, were considered. 
Table 1

Demographic data of patients according to revascularization types.

\begin{tabular}{|c|c|c|c|}
\hline & $\begin{array}{l}\text { IR group } \\
(32)\end{array}$ & $\begin{array}{l}\text { CR group } \\
\text { (58) }\end{array}$ & $p$ value \\
\hline Male gender & $31(96.9 \%)$ & $53(91.4 \%)$ & 0.320 \\
\hline Age (years) & $59.6 \pm 9.8$ & $60.5 \pm 10.6$ & 0.704 \\
\hline DM & $15(46.9 \%)$ & $31(53.4 \%)$ & 0.553 \\
\hline DM with end-organ damage & $1(3.1 \%)$ & $2(3.4 \%)$ & 0.935 \\
\hline CKD-4,5 & $2(6.2 \%)$ & $7(12.1 \%)$ & 0.381 \\
\hline NYHA-3,4 & $5(15.6 \%)$ & $6(10.3 \%)$ & 0.467 \\
\hline COPD & $0(0.0 \%)$ & $0(0.0 \%)$ & 1.000 \\
\hline PAOD & $0(0.0 \%)$ & $4(6.9 \%)$ & 0.131 \\
\hline Old CVA or TIA & $3(9.4 \%)$ & $3(5.2 \%)$ & 0.447 \\
\hline Malignancy & $0(0.0 \%)$ & $5(8.6 \%)$ & 0.089 \\
\hline $\mathrm{CCl}$ & $1.34 \pm 1.54$ & $1.45 \pm 1.78$ & 0.780 \\
\hline EuroScore II & $0.28 \pm 0.15$ & $0.24 \pm 0.12$ & 0.155 \\
\hline $\mathrm{OHCA}$ & $11(34.4 \%)$ & $30(51.7 \%)$ & 0.116 \\
\hline Initial shockable rhythm (VT, VF) & $23(71.9 \%)$ & $39(67.2 \%)$ & 0.651 \\
\hline Low-flow duration (min) & $43.0 \pm 19.1$ & $42.8 \pm 20.8$ & 0.963 \\
\hline CK-MB on admission (ng/mL) & $158.0 \pm 250.6$ & $88.6 \pm 169.7$ & 0.156 \\
\hline \multicolumn{4}{|l|}{ Coronary lesions } \\
\hline LM & $9(28.1 \%)$ & $12(20.7 \%)$ & 0.427 \\
\hline LAD & $30(93.7 \%)$ & $43(74.1 \%)$ & $0.024^{\star \star}$ \\
\hline LCX & $29(90.6 \%)$ & $31(53.4 \%)$ & $<0.001^{\star *}$ \\
\hline RCA & $27(84.4 \%)$ & $34(58.6 \%)$ & $0.013^{\star \star}$ \\
\hline Initial ischemic regions & $2.7 \pm 0.5$ & $1.9 \pm 0.9$ & $<0.001 * \star$ \\
\hline IABP use & $28(87.5 \%)$ & $42(72.4 \%)$ & 0.101 \\
\hline $\mathrm{PCl}$ & $26(81.2 \%)$ & $40(69.0 \%)$ & 0.210 \\
\hline CABG & $6(18.8 \%)$ & $19(32.8 \%)$ & 0.158 \\
\hline
\end{tabular}


IR group

(32)
CR group

(58)

AMI: acute myocardial infarct, CCl: Charlson comorbidity index, CK-MB: creatine kinase MB isoenzyme, CKD: chronic kidney disease, COPD: chronic obstructive pulmonary disease, CPR: cardiopulmonary resuscitation, PR: partial revascularization, CVA: cerebral vascular attack, DM: diabetes mellitus, ECMO: extracorporeal membrane oxygenation, IABP: intra-aortic balloon pumping, NYHA: New-York Heart association, OHCA: out-of-hospital cardiac arrest, PAOD: peripheral artery occlusive disease TIA: transient ischemic attack, CR: complete revascularization, UR: unsuccessful revascularization, VF: ventricular fibrillation, VT: ventricular tachycardia.

Initial CAD ischemic regions are counted by the number of involvements of left anterior descending (LAD), left circumflex (LCX), right coronary artery (RCA), with left main (LM) is viewed as involvement of LAD and LCX. PCl: percutaneous coronary intervention, including balloon angioplasty or stenting.

Low-flow duration: duration between CPR and ECMO run.

* denotes a trend of statistical significance. ** denotes statistical significance.

Emergency $\mathrm{PCl}$ was performed using the femoral artery approach. The coronary lesions were first prepared through balloon angioplasty followed by stent implantation. The activating clotting time was maintained in the range of 250-300 s through continuous heparin infusion; intracoronary $\mathrm{llb} / \mathrm{llla}$ inhibitors or aspiration catheter were administered if the thrombus burden was large or a TIMI 3 flow was not obtained. Those patients with post-PCI TIMI 1 or 2 flow or with coronary anatomy inappropriate for $\mathrm{PCl}$, emergency CABG was performed soon with ECMO support. Side-clamping of the ascending aorta was applied for saphenous vein graft anastomosis to the ascending aorta in most cases. Anastomosis of target coronary arteries was performed under a beating heart condition in most patients. Induced cardiac arrest with cardioplegia was used in patients with difficult exposure of target vessels or very hemorrhagic and fragile myocardium.

\section{Exclusion criteria}

The patients who experienced cardiac arrest and received CPR $<10$ min before the return of spontaneous circulation (ROSC) were excluded from the study because they did not fulfil the criteria of prolonged CPR. Patients with acute aortic dissection, massive intracranial hemorrhage, prosthetic valve dysfunction, endocrine crisis, or failure of ROSC after ECMO support were excluded from further coronary intervention. Patients who received CPR and ECMO after coronary intervention or CABG during the same admission were excluded. Transferred patients who did not receive their index ECPR in the study hospital were also excluded because of unstandardized definitions of arrest time, CPR duration, and ECMO management protocol. Patients who experienced shock necessitating ECMO in an elective or emergency situation without cardiac massage were also excluded from the study. Patients with clinical presentation of malignant arrhythmia with patent coronary arteries were excluded. Patients refused to receive $\mathrm{PCl}$ or CABG after coronary angiography study were also excluded from the study. Patients with incomplete data of CPR, follow-up, and coronary angiography were also excluded. 
ECMO equipment and management has been described elsewhere. $(5,6)$ The principal components of the ECMO circuit used on our patients were centrifugal pumps and an oxygenator (Medtronic, Anaheim, CA, USA; Medos, Stolberg, Germany; Maquet, Rastatt, Germany). The circuit was pre-assembled without priming and was then primed using saline containing $2 \mathrm{U} / \mathrm{mL}$ heparin on receiving an ECPR call.

Review of coronary angiography data to determine revascularization types was retrospectively performed by two independent cardiologists (L.Y.L. and W.Y.C.). Coronary artery disease was defined as coronary vessels with stenosis $>70 \%$ or left main vessel $>50 \%$ stenosis by simple visual estimation. Complete revascularization (CR) was defined by each of the involved territories (left anterior descending [LAD], left circumflex [LCX], and right coronary artery) being successfully revascularized through PCI or CABG 24 hours after the index CPR episode. Incomplete revascularization (IR) was defined as culprit vessel only intervention or culprit vessel plus another artery intervention, leaving at least one diseased coronary artery territory. Successful coronary angioplasty, through either balloon angioplasty or coronary stenting, was defined as a TIMI 3 flow at the end of the emergency procedure. When left main coronary artery is involved, the patient is considered to have a two-vessel disease (LAD or LCX.)

\section{Outcomes}

The primary endpoint of the study was a favorable neurological outcome (Cerebral Performance Category [CPC] 1 or 2) at hospital discharge. The second endpoint is the composite of major adverse cardiac and cerebral events (MACCE) defined as cardiac death, myocardial infarction (MI), revascularization, or stroke at 1 year since the index ECPR. Successful weaning of ECMO is defined as separation from ECMO support without subsequent mortality in the next $48 \mathrm{~h}$. Safety endpoints were adverse events of infection, limb ischemia, renal failure, bleeding, or stroke during hospitalization among different study groups. The follow-up data of the patients who survived ECPR to hospital discharge were collected through chart review.

\section{Statistical analysis}

All demographic data and post-ECMO events data were prospectively collected from an ECMO registration database, from which several studies regarding ECPR had been reported.(5) ${ }^{-}(7)$ (8) Continuous variables are presented as mean \pm standard deviation and were examined using the Student $t$ test and analysis of variance (ANOVA). Categorical variables were analyzed using the chi-square test or Fisher exact test, depending on sample sizes. The variables affecting the major outcomes (i.e., hospital survival and favorable neurological function) that were significant $(P<0.15)$ in the univariate analysis were included in multivariate logistic regression with backward stepwise analysis. The patients' 1-year survival was modeled using the Kaplan-Meier method and tested using the log-rank test and Cox regression. Statistical significance was set at $P<0.05$ and statistical significance was set at $0.10>P>$ 0.05. All statistical analyses were performed using MedCalc (version 17.8.6; MedCalc Software, Ostend, 
Belgium). All statistical analyses were reviewed by a specialist (J.W.L) blinded to the present study design.

\section{Results}

A total of 502 adult ECPR patients during the study period was retrieved from a prospectively collected database and retrospectively analyzed. Among the patients, 115 patients were suspected with acute coronary syndrome and underwent emergency coronary angiography study. After excluding the patients who did not receive revascularization procedure (13), with patent coronary arteries (7), or with incomplete angiography data (5), 90 patients were included in the present study (Fig. 1). Among those patients, 39 received successful $P C I$ to achieve $C R, 26$ received $P C I$ to achieve IR, 9 received emergent CABG following primary PCl with TIMI 2 flow in target-vessel, 16 received CABG without $\mathrm{PCl}$. Among the 25 patients who received CABG, 19 and 6 achieved CR and IR, respectively. Therefore, 58 patients of CR and 32 patients of IR were included into the analysis. The number of patients achieving CR increased gradually from 2006 to 2016 (22, 30, and 38 patients in 2006-2009, 2010-2013, and 2014-2016, respectively), and the percentage of $\mathrm{CR}$ also increased in consecutive periods $(40.9 \%, 70.0 \%$, and $73.7 \%$, respectively, $\mathrm{P}=0.028)$. (Supplement Fig. 1).

The demographic data of both study groups are presented in Table 1. In brief, most demographic data are comparable between IR and CR groups except the extent of coronary artery involvement. The number of ischemic territories was higher in IR group $(2.7 \pm 0.5)$ than that in $C R$ group $(1.9 \pm 0.9)(P, 0.001)$. Regarding the intervention strategy, $69.0 \%$ of IR and $81.2 \%$ of $C R$ received $P C I(P=0.210)$, and $18.8 \%$ of IR and $32.8 \%$ of $C R$ received CABG $(P=0.158$.) The average graft number for CABG cases $(25)$ was $3.0 \pm$ 0.8 .

The safety endpoints among the different study groups are listed in Table 2. The incidence of infection, limb ischemia needing fasciotomy or amputation, renal failure needing mechanical renal replacement therapy, brain anoxia, stroke (ischemic or hemorrhagic), and bleeding are all comparable among the three groups. This finding indicates that CR group, even with more CABG procedure than IR group, is not associated with increased risk of peri-procedural morbidity. It is noteworthy that ischemic stroke rate is higher in CR group (12.1\%) as compared with IR group (3.1\%), even though which does not achieve statistical significance $(P=0.156$. $)$ 
Table 2

ECPR outcome

\begin{tabular}{|c|c|c|c|}
\hline & $\begin{array}{l}\text { IR group } \\
\text { (32) }\end{array}$ & $\begin{array}{l}\text { CR group } \\
\text { (58) }\end{array}$ & $p$ value \\
\hline \multicolumn{4}{|l|}{ Safety endpoints } \\
\hline Infection-all & $10(31.2 \%)$ & $17(29.3 \%)$ & 0.848 \\
\hline Infection-wound & $1(3.1 \%)$ & $5(8.6 \%)$ & 0.320 \\
\hline Infection-pneumonia & $3(9.4 \%)$ & $8(13.8 \%)$ & 0.542 \\
\hline Infection-bacteremia & $6(18.8 \%)$ & $6(10.3 \%)$ & 0.264 \\
\hline Limb ischemia & $2(6.2 \%)$ & $5(8.6 \%)$ & 0.689 \\
\hline MRRT & $13(40.6 \%)$ & $20(34.5 \%)$ & 0.565 \\
\hline Brain anoxia & $12(37.5 \%)$ & $19(32.8 \%)$ & 0.652 \\
\hline CVA ischemic & $1(3.1 \%)$ & $7(12.1 \%)$ & 0.156 \\
\hline CVA hemorrhagic & $0(0.0 \%)$ & $1(1.7 \%)$ & 0.458 \\
\hline Bleeding & $5(15.6 \%)$ & $13(22.4 \%)$ & 0.443 \\
\hline \multicolumn{4}{|l|}{ Hospital outcome } \\
\hline ECMO days & $4.1 \pm 3.7$ & $4.1 \pm 3.0$ & 0.989 \\
\hline Ventilator days & $12.9 \pm 15.9$ & $12.1 \pm 14.7$ & 0.807 \\
\hline ICU days & $13.6 \pm 17.0$ & $12.8 \pm 13.1$ & 0.802 \\
\hline Hospitalization days & $20.3 \pm 27.3$ & $21.1 \pm 22.7$ & 0.785 \\
\hline Weaned off ECMO & $14(43.7 \%)$ & $36(62.1 \%)$ & $0.096 *$ \\
\hline Weaned off ECMO without VAD or HTX & $14(43.7 \%)$ & $35(60.3 \%)$ & 0.132 \\
\hline Hospital survival at discharge & $9(28.1 \%)$ & $28(48.3 \%)$ & $0.064^{*}$ \\
\hline Favorable neurological outcome $(\mathrm{CPC} 1,2)$ at discharge & $7(21.9 \%)$ & $22(37.9 \%)$ & 0.121 \\
\hline LVEF at hospital discharge (\%), (survivors) & $44.3 \pm 7.6$ & $48.2 \pm 13.0$ & 0.416 \\
\hline \multicolumn{4}{|c|}{$\begin{array}{l}\text { CPC: Cerebral performance category, HTX: heart transplantation, LVEF: left ventricular ejection } \\
\text { fraction, MRRT: mechanical renal replacement therapy, VAD: ventricular assist device. }\end{array}$} \\
\hline
\end{tabular}

Hospital outcomes are also summarized in Table 2. In brief, ECMO days, ventilator days, days of intensive care unit, and hospitalization days were comparable among both groups. One patient received ventricular 
assist device (VAD) support and another one received heart transplantation (HTX). The rate of successful wean-off of ECMO without VAD or HTX was higher in the CR (60.3\%) than that in IR (43.7\%) but not attain statistical significance $(P=0.132)$. Hospital survival is also marginally higher in CR $(48.3 \%)$ than that in IR $(28.1 \%)(P=0.064)$. Favorable neurological outcome at hospital discharge $(C P C 1,2)$ was also higher in CR group (37.9\%) than in IR group (21.9\%), but it does not achieve statistic significance $(P=0.121)$ (Table 2).

Logistic regression analysis of favorable neurological outcome at hospital discharge is presented in Table 3. The univariate logistic regression analysis revealed that only age is a potentially significant factor (odds ratio [OR] for every 10 -year increase in age is $0.66, P=0.078$ ). The low-flow duration, $C A B G$, or $\mathrm{CR}$ are not shown as significant risk factors. Multivariate logistic regression is not performed because only one factor (age) is found by univariate logistic regression analysis.

Table 3

Logistic regression analysis for hospital survival with favorable neurological functions (CPC 1,2).

\begin{tabular}{|lllll|}
\hline & Univariate analysis & & Multivariate analysis \\
\hline & OR $(95 \% \mathrm{Cl})$ & $\mathrm{P}$ & OR $(95 \% \mathrm{Cl})$ & $\mathrm{P}$ \\
\hline Female gender & $1.06(0.18-6.12)$ & 0.952 & & \\
\hline Age (+ 10 years) & $0.66(0.41-1.05)$ & $0.078^{*}$ & $0.66(0.41-1.05)$ & 0.078 \\
\hline CCI & $1.00(0.77-1.30)$ & 0.992 & \\
\hline EuroScore II & $0.16(0.00-6.17)$ & 0.323 & \\
\hline Low-flow duration (+ 10 min) & $0.83(0.65-1.07)$ & 0.148 & \\
\hline OHCA & $0.51(0.20-1.27)$ & 0.149 & \\
\hline Initial shockable rhythm (VT, VF) & $0.61(0.22-1.65)$ & 0.327 & \\
\hline Initial ischemic regions & $0.79(0.46-1.35)$ & 0.394 & \\
\hline CABG & $0.99(0.37-2.65)$ & 0.978 & \\
\hline IABP & $2.04(0.74-5.69)$ & 0.170 & \\
\hline Complete Revascularization (CR vs. IR) & $1.82(0.70-4.77)$ & 0.221 & \\
\hline $\begin{array}{l}\text { Variables with P< 0.15 by univariate analysis (marked with an asterisk) were included in multivariate } \\
\text { analysis. The multivariate logistic regression analysis was set with entry and removal P values of } \\
0.10 \text { and 0.15, respectively. }\end{array}$ & & \\
\hline
\end{tabular}

In 1-year follow-up of the hospital survivors, mortality was recorded in four cases, all attributable to cardiac causes (one in IR and three in CR groups.) Morbidity was recorded as following: two patients received PCI (one each in IR and CR groups), one received CABG (in the IR group), and one had stroke (in IR group). In total, six cases with MACCE in 1-year follow-up period (three in IR and three in CR groups.) 
Kaplan-Meier survival analysis for 1-year freedom from MACCE probability for both study groups are illustrated in Fig. 2, revealing marginally higher probability in CR group than in IR groups (42.4\% for CR; $24.6 \%$ for $I R, P=0.051$ ). Taking only hospital survivors into consideration, the freedom from MACCE probability at 1 -year is comparable between CR group (87.5\%) and IR group (67.5\%) $(P=0.162$.)

Cox regression analysis for 1-year MACCE probability for all study patients is presented in Table 4. Univariate analysis revealed that age, Euroscore II, low-flow duration, initial shockable rhythm, CAD number, and CR were potential significant factors. In multivariate analysis, only age, low-flow duration, initial shockable rhythm, and CR were revealed as significant factors (hazard ratios: 1.37 for every 10-year increase in age, 1.23 every 10-minute increase in low-flow duration, 0.53 for initial shockable rhythm, and 0.53 for $C R ; P=0.002,0.027,0.027$, and 0.020 , respectively).

Table 4

Cox regression analysis for 1-year MACCE probability.

\begin{tabular}{|c|c|c|c|c|}
\hline & \multicolumn{2}{|l|}{ Univariate analysis } & \multicolumn{2}{|c|}{ Multivariate analysis } \\
\hline & $\mathrm{HR}(95 \% \mathrm{Cl})$ & $\mathrm{P}$ & $\mathrm{HR}(95 \% \mathrm{Cl})$ & $P$ \\
\hline Female gender & $1.03(0.37-2.85)$ & 0.954 & & \\
\hline Age $(+10$ years $)$ & $1.38(1.08-1.76)$ & $0.012 * \star$ & $1.37(1.05-1.79)$ & $0.022 * \star$ \\
\hline $\mathrm{CCl}$ & $1.08(0.94-1.24)$ & 0.302 & & \\
\hline EuroScore II & $6.51(1.11-38.2)$ & $0.046^{* \star}$ & -- & N.S. \\
\hline Low-flow duration (+ $10 \mathrm{~min}$ ) & $1.20(1.05-1.38)$ & $0.008 * \star$ & $1.23(1.08-1.42)$ & $0.002^{\star \star}$ \\
\hline $\mathrm{OHCA}$ & $0.95(0.57-1.59)$ & 0.844 & & \\
\hline Initial shockable rhythm (VT, VF) & $0.60(0.35-1.02)$ & $0.064^{*}$ & $0.53(0.31-0.93)$ & $0.027 * \star$ \\
\hline CAD numbers & $1.36(0.99-1.87)$ & $0.053 * \star$ & - & N.S. \\
\hline CABG & $1.04(0.60-1.82)$ & 0.880 & & \\
\hline IABP & $1.44(0.75-2.77)$ & 0.261 & & \\
\hline CR vs. IR & $0.61(0.37-1.02)$ & $0.066^{*}$ & $0.53(0.31-0.91)$ & $0.020 * *$ \\
\hline \multicolumn{5}{|c|}{$\begin{array}{l}\text { Variables with } P<0.10 \text { by univariate analysis (marked with asterisk) were put to multivariate analysis. } \\
\text { The multivariate logistic regression analysis was set with entry and removal } P \text { values of } 0.10 \text { and } \\
0.15 \text {, respectively. NS: nonsignificant. HR: hazard ratio }\end{array}$} \\
\hline
\end{tabular}

\section{Discussion}

The present study is the largest series focusing on the impact of revascularization strategy for AMI patients complicated with cardiac arrest under ECMO support. The results indicated that CR patients is associated with insignificantly higher rate of favorable neurological outcomes at hospital discharge, and 
lower MACCE probabilities at 1-year follow-up than IR patients. The results might contribute to the decision-making in revascularization strategy in this extremely critical condition.

AMI complicated with cardiogenic shock is a highly emergent condition needing immediate decision to support the critical hemodynamics and protect the jeopardized myocardium.(9)'(10) Data from IABPSHOCK II trial revealed a 30 -day mortality of $43.6 \%$ in patients with AMI complicated by cardiogenic shock with or without IABP support.(11) Several studies have advocated ECMO use during primary PCI for AMI with cardiogenic shock.(12)'(13) Studies have also advocated primary PCI for patients with AMI after ECMO use to improve hospital survival rates.(14) The mortality reported in the present study was considerably higher than that reported in the SHOCK I or IABP-SHOCK II trials because the present study group comprised a relatively severe patients in dying condition. However, the present study outcomes were comparable with those of our previous studies on ECPR.(4) ${ }^{-}(6)^{-} \cdot(7)$

Previous study(15) had reported a systemic approach to OHCA patients, including ECMO initiation and immediate coronary angiography in patients with presumed ischemic etiology increased the rate of favorable neurological outcomes at hospital discharge from 3.3\% in 2009 to $8.5 \%$ in 2013. However, the types of coronary revascularization in that study was not reported in detail. Other studies have suggested infarct-related $\mathrm{PCl}$ (e.g., HORIZONS-AMI study,(16) APEX-AMI trial(17)) in patients with AMI without cardiogenic shock, whereas others have suggested multivessel PCI (e.g., SWISSI II trial(18) and HELP$\operatorname{AMI}(19))$. The CULPRIT-SHOCK trial demonstrated that in patients with AMI complicated by cardiogenic shock, immediate culprit-lesion-only PCl had better clinical outcomes than immediate multivessel $\mathrm{PCl}$ in patients with AMI and cardiogenic shock.(20)'(21) However, the CULPRIT-SHOCK trial did not include patients with prolonged resuscitation.(20) The results of a 1-year follow-up study of the CULPRIT-SHOCK trial(21) showed that although the mortality rates did not differ significantly between the culprit-lesiononly and multivessel PCl groups, the incidence of rehospitalization and repeated intervention was considerably higher in the culprit-lesion-only $\mathrm{PCl}$ group than in the multivessel $\mathrm{PCl}$ group during the follow-up. This finding indicates that for patients with AMI without intractable cardiogenic shock, the benefit of solving all residual myocardial ischemic burdens does not outweigh the risk of the procedure, but it needs to be treated during the follow-up period. Theoretically, the presence of silent myocardial ischemic burden is associated with a negative effect on long-term outcome. ${ }^{21-23}$ The necessity to treat residual myocardial ischemic burden is supported by other meta-analysis,(22) in which during primary $\mathrm{PCl}$ in patients with $\mathrm{AMI}$ without cardiogenic shock, $\mathrm{CR}$ was associated with a significantly lower total mortality and MI risk during follow-up than PR was. In addition, a post hoc analysis of the IABP-SHOCK II trial(23) indicated that the presence of chronic total occlusion in a non-infarction-related vessel is associated with an increased ventricular arrhythmia incidence and mortality at 12-month follow-up after index AMI. However, whether non-infarct-related myocardial ischemic burden needs to be immediately treated in detrimental AMI complicated with cardiac arrest needing ECMO support is unanswered in those studies. From the results of the present study, considering that CR did not increase the early mortality in this group, we suggest $\mathrm{CR}$ for the patients to achieve the best chance of hospital survival with favorable neurological status and high 1-year freedom from MACCE probability.

Page $12 / 20$ 
Regarding the procedural risk and complications, data of the present study indicate that CR group, even with more CABG procedure than IR group, is not associated with increased risk of peri-procedural morbidity. It is noteworthy that ischemic stroke rate is higher in CR group (12.1\%) as compared with IR group (3.1\%), even though which does not achieve statistical significance $(P=0.156$.) Considering that emergency more CABG was performed in CR group with ECMO support as compared with IR group, and using saphenous vein grafts in most cases, the high incidence of ischemic stroke might be explained by inadequate cardiac support by ECMO during exposure of the posterior ventricle in the CABG operation, and embolization during side-clamping of the ascending aorta during anastomosis of the proximal vein grafts. In addition, early studies indicates that the incidence of postoperative pneumonia was also higher in CABG patients as compared with other types of operation. $(24,25)$ This high incidence of post-CABG pneumonia can be explained by prolonged pump time, excessive blood transfusion, prolonged ICU stay, and limited motility due to IABP and ECMO use, all of which are associated with risk factors for postoperative pneumonia.(24)

Low-flow duration has generally been recognized as a significant risk factor for neurological outcome(7)' $(26,27)(8)$ but not in the present study. The possible explanation is a relatively short low-flow duration (42.4 $\pm 19.1 \mathrm{~min})$ in the present study. Compared with several studies focusing on ECPR for patients with satisfactory clinical outcomes, $(1,28)$ the median low-flow duration for the survivor group was similar at $40 \mathrm{~min}$. By the satisfactory hospital survival rates from both the CHEER trial (53.8\%) and present study (38.8\%), we suggest that special CPR efforts are worthy for cardiac arrest patients with presenting symptoms or initial EKG suspecting AMI as the triggering cause.

Regarding IABP use or not in AMI with shock, our results reconfirmed the neutral effect of IABP in the IABP-SHOCK II trial.(10)'(11) The multivariate logical regression analysis in the present study did not demonstrate the beneficial effect of IABP on hospital survival rate, in accordance with previous studies' findings.(29)

\section{Limitations}

Therapeutic hypothermia was not routinely applied in the patients because ECMO systemic hypothermia is not a routine procedure in our institute. Whether therapeutic hypothermia is beneficial to neurological outcome for patients with cardiac arrest remains controversial and is under investigation.(1) $)^{(30)^{-}(31)}$ Several strategies for systemic or local hypothermia, such as cerebral selective deep hypothermia after ECPR(32) are under currently investigation in our institute.

EKG was not obtainable for all patients during the ECPR condition to clearly define ST-elevation MI (STEMI) or non-STEMI. However, according to their severity and emergency, the treatment protocol was based on the guideline for STEMI.

The present study was not a randomized trial. However, the decision of revascularization strategy was made by a joint discussion between on-duty cardiologists and cardiac surgeons. It may be considered as 
real-world data reflecting the true clinical scenario.

\section{Conclusion}

For AMI patients complicated with cardiac arrest supported with ECMO, CR, as compared with IR, had similar outcome in one-year MACCE outcomes. Therefore, we suggest culprit strategy may be acceptable for these severe AMI patients under ECMO support in the point of saving procedure duration.

\section{Declarations}

\section{Funding}

The study was partially supported by the grant from 106-2314-B-002 -234 -MY3, 102-2325-B-009, Ministry of Science and Technology, Taiwan.

\section{Competing interest}

Chen YS was a receipt of the grant of 106-2314-B-002 -234 -MY3, 102-2325-B-009, Ministry of Science and Technology, Taiwan.

The other authors (Yu HY, Wang CH, Lin LY, Lin JW, Chi NH, Wang YC, Chou HW, Chou NK, Hwang JJ) received no financial support for the research, authorship, and/or publication of this article.

\section{Ethical Approval and consent to participate}

All demographic and clinical data were prospectively collected and systemically reviewed under institutional approval (NTUH-9561707007.) The present study was approved by the review board of our institute (NTUH 201810079 RIN).

\section{Consent for publication}

All authors have read and agreed to publish the manuscript and the data

\section{Availability of supporting data}

The supplement information was submitted, and please refer to the supplement.

\section{Authors' contribution}

Concept formation: $Y u H Y$, Chen YS

Data collection: Wang $\mathrm{CH}$, Chi NH, Wang YC, Chou HW, Chou NK, Manuscript writing: Yu HY, Wang YC, Chou NK 
Statistical analysis:Lin LY

Statistical reviewing: Lin JW,

Critical editing and reviewing: Hwang JJ, Chen YS

\section{Acknowledgement}

The study was partially supported by the research fund of the Ministry of Science and Technology, Taiwan (MOST 106-2314-B-002-154-MY3 and MOST 106-2314-B-002-234-MY3).

\section{References}

1. Stub D, Bernard S, Pellegrino V, et al. Refractory cardiac arrest treated with mechanical CPR, hypothermia, ECMO and early reperfusion (the CHEER trial). Resuscitation. 2015;86:88-94.

2. Belohlavek J, Kucera K, Jarkovsky J, et al. Hyperinvasive approach to out-of hospital cardiac arrest using mechanical chest compression device, prehospital intraarrest cooling, extracorporeal life support and early invasive assessment compared to standard of care. A randomized parallel groups comparative study proposal. "Prague OHCA study". J Transl Med. 2012;10:163.

3. Ibanez B, James S, Agewall S, et al. 2017 ESC Guidelines for the management of acute myocardial infarction in patients presenting with ST-segment elevation: The Task Force for the management of acute myocardial infarction in patients presenting with ST-segment elevation of the European Society of Cardiology (ESC). Eur Heart J. 2018;39:119-77.

4. Chen Y-S, Lin J-W, Yu H-Y, et al. Cardiopulmonary resuscitation with assisted extracorporeal lifesupport versus conventional cardiopulmonary resuscitation in adults with in-hospital cardiac arrest: an observational study and propensity analysis. Lancet. 2008;372:554-61.

5. Wang C-H, Chou N-K, Becker LB, et al. Improved outcome of extracorporeal cardiopulmonary resuscitation for out-of-hospital cardiac arrest-a comparison with that for extracorporeal rescue for in-hospital cardiac arrest. Resuscitation. 2014;85:1219-24.

6. Chen Y-S, Chao A, Yu H-Y, et al. Analysis and results of prolonged resuscitation in cardiac arrest patients rescued by extracorporeal membrane oxygenation. JAC. 2003;41:197-203.

7. Tseng L-J, Yu H-Y, Wang C-H, et al. Impact of Age-Adjusted Charlson Comorbidity on Hospital Survival and Short-Term Outcome of Patients with Extracorporeal Cardiopulmonary Resuscitation. J Clin Med 2018;7.

8. Yu H-Y, Wang C-H, Chi N-H, et al. Effect of interplay between age and low-flow duration on neurologic outcomes of extracorporeal cardiopulmonary resuscitation. Intensive Care Med. 2018;63:60-11.

9. Hochman JS, Sleeper LA, Webb JG, et al. Early revascularization in acute myocardial infarction complicated by cardiogenic shock. SHOCK Investigators. Should We Emergently Revascularize Occluded Coronaries for Cardiogenic Shock. N Engl J Med. 1999;341:625-34. 
10. Thiele H, Zeymer U, Neumann F-J, et al. Intraaortic balloon support for myocardial infarction with cardiogenic shock. N Engl J Med. 2012;367:1287-96.

11. Thiele H, Zeymer U, Neumann F-J, et al. Intra-aortic balloon counterpulsation in acute myocardial infarction complicated by cardiogenic shock (IABP-SHOCK II): final 12 month results of a randomised, open-label trial. Lancet. 2013;382:1638-45.

12. Sheu J-J, Tsai T-H, Lee F-Y, et al. Early extracorporeal membrane oxygenator-assisted primary percutaneous coronary intervention improved 30-day clinical outcomes in patients with ST-segment elevation myocardial infarction complicated with profound cardiogenic shock. Crit Care Med. 2010;38:1810-7.

13. Sakamoto S, Taniguchi N, Nakajima S, Takahashi A. Extracorporeal life support for cardiogenic shock or cardiac arrest due to acute coronary syndrome. The Annals of Thoracic Surgery. 2012;94:17.

14. Wu M-Y, Tseng Y-H, Chang Y-S, Tsai F-C, Lin PJ. Using extracorporeal membrane oxygenation to rescue acute myocardial infarction with cardiopulmonary collapse: The impact of early coronary revascularization. Resuscitation. 2013;84:940-5.

15. Hwang WS, Park JS, Kim SJ, Hong YS, Moon SW, Lee SW. A system-wide approach from the community to the hospital for improving neurologic outcomes in out-of-hospital cardiac arrest patients. Eur J Emerg Med. 2017;24:87-95.

16. Kornowski R, Mehran R, Dangas G, et al. Prognostic impact of staged versus "one-time" multivessel percutaneous intervention in acute myocardial infarction: analysis from the HORIZONS-AMI (harmonizing outcomes with revascularization and stents in acute myocardial infarction) trial. J Am Coll Cardiol. 2011;58:704-11.

17. Toma M, Buller CE, Westerhout CM, et al. Non-culprit coronary artery percutaneous coronary intervention during acute ST-segment elevation myocardial infarction: insights from the APEX-AMI trial. Eur Heart J. 2010;31:1701-7.

18. Erne P, Schoenenberger AW, Burckhardt D, et al. Effects of percutaneous coronary interventions in silent ischemia after myocardial infarction: the SWISSI II randomized controlled trial. JAMA. 2007;297:1985-91.

19. Di Mario C, Mara S, Flavio A, et al. Single vs multivessel treatment during primary angioplasty: results of the multicentre randomised HEpacoat for cuLPrit or multivessel stenting for Acute Myocardial Infarction (HELP AMI) Study. Int J Cardiovasc Intervent. 2004;6:128-33.

20. Thiele H, Akin I, Sandri M, et al. PCI Strategies in Patients with Acute Myocardial Infarction and Cardiogenic Shock. N Engl J Med. 2017;377:2419-32.

21. Thiele H, Akin I, Sandri M, et al. One-Year Outcomes after PCI Strategies in Cardiogenic Shock. N Engl J Med. 2018;379:1699-710.

22. Pasceri V, Patti G, Pelliccia F, et al. Complete Revascularization During Primary Percutaneous Coronary Intervention Reduces Death and Myocardial Infarction in Patients With Multivessel Disease: 
Meta-Analysis and Meta-Regression of Randomized Trials. JACC: Cardiovascular Interventions 2018;11:833-843.

23. Saad M, Fuernau G, Desch S, et al. Prognostic impact of non-culprit chronic total occlusions in infarct-related cardiogenic shock: results of the randomised IABP-SHOCK II trial. Eurolntervention. 2018;14:e306-13.

24. Kilic A, Ohkuma R, Grimm JC, et al. A novel score to estimate the risk of pneumonia after cardiac surgery. The Journal of Thoracic Cardiovascular Surgery. 2016;151:1415-20.

25. Allou N, Bronchard R, Guglielminotti J, et al. Risk factors for postoperative pneumonia after cardiac surgery and development of a preoperative risk score*. Crit Care Med. 2014;42:1150-6.

26. Wengenmayer T, Rombach S, Ramshorn F, et al. Influence of low-flow time on survival after extracorporeal cardiopulmonary resuscitation (eCPR). Crit Care. 2017;21:157.

27. Debaty G, Babaz V, Durand M, et al. Prognostic factors for extracorporeal cardiopulmonary resuscitation recipients following out-of-hospital refractory cardiac arrest. A systematic review and meta-analysis. Resuscitation. 2017;112:1-10.

28. Han SJ, Kim HS, Choi HH, et al. Predictors of survival following extracorporeal cardiopulmonary resuscitation in patients with acute myocardial infarction-complicated refractory cardiac arrest in the emergency department: a retrospective study. J Cardiothorac Surg. 2015;10:23.

29. Liao LINL-Y, Wang C-W. C-H, et al. Effects of Additional Intra-aortic Balloon Counter-Pulsation Therapy to Cardiogenic Shock Patients Supported by Extra-corporeal Membranous Oxygenation. Sci Rep. 2016;6:23838.

30. Nielsen $\mathrm{N}$, Wetterslev J, Cronberg T, et al. Targeted temperature management at $33^{\circ} \mathrm{C}$ versus $36^{\circ} \mathrm{C}$ after cardiac arrest. N Engl J Med. 2013;369:2197-206.

31. Stammet P, Dankiewicz J, Nielsen N, et al. Protein S100 as outcome predictor after out-of-hospital cardiac arrest and targeted temperature management at $33^{\circ} \mathrm{C}$ and $36^{\circ} \mathrm{C}$. Crit Care. 2017;21:153.

32. Wang C-H, Lin Y-T, Chou H-W, et al. Novel approach for independent control of brain hypothermia and systemic normothermia: cerebral selective deep hypothermia for refractory cardiac arrest. J Neurointerv Surg. 2017;9:e32.

\section{Figures}




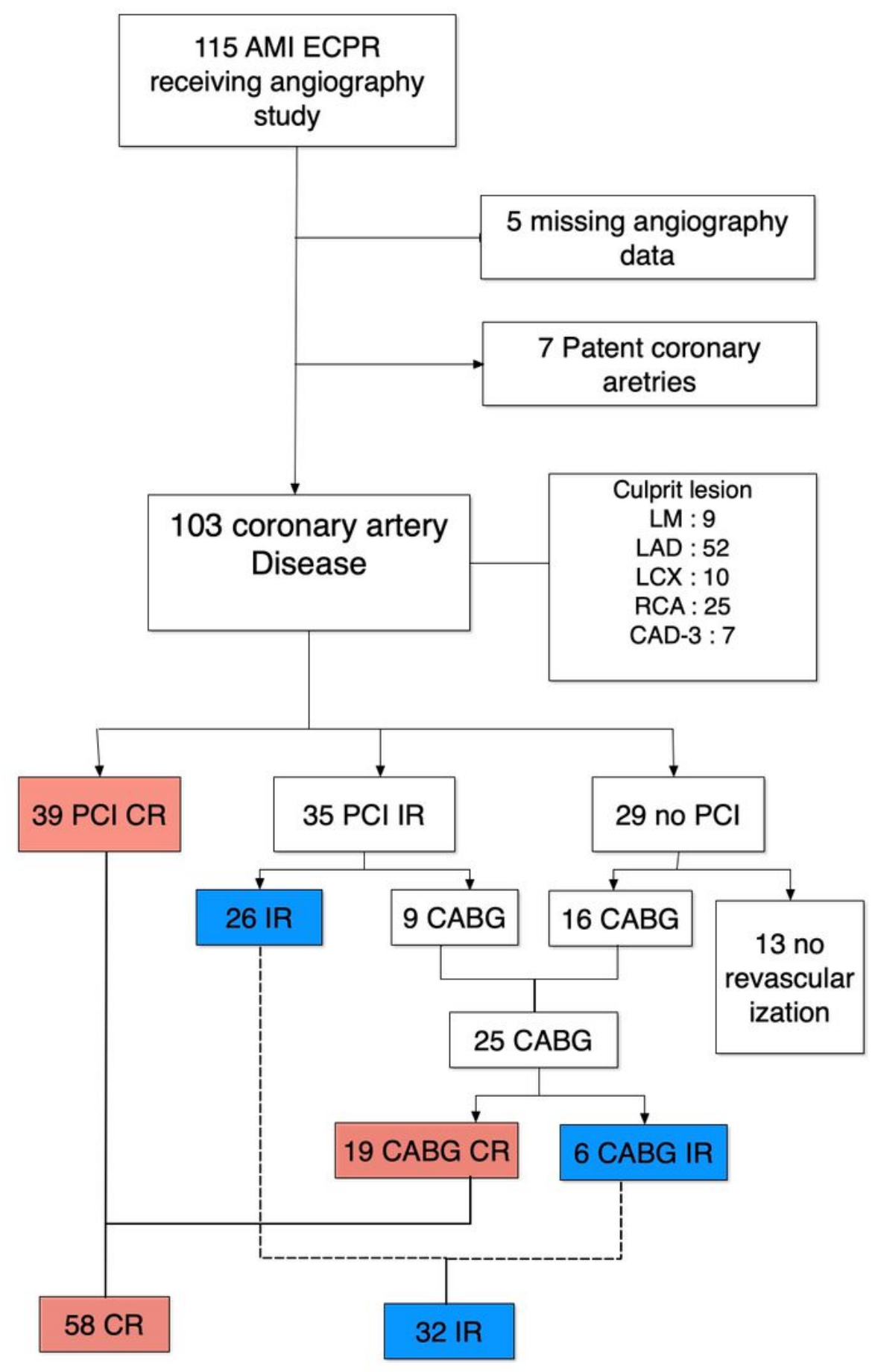

\section{Figure 1}

Flowchart of the study patient. Legend: CABG: coronary artery bypass surgery,CR: complete revascularization,ECPR: extracorporeal cardiopulmonary resuscitation, IR: incomplete revascularization,LAD: left anterior descending, LCX: left circumflex, LM: left main, RCA: right coronary artery. 


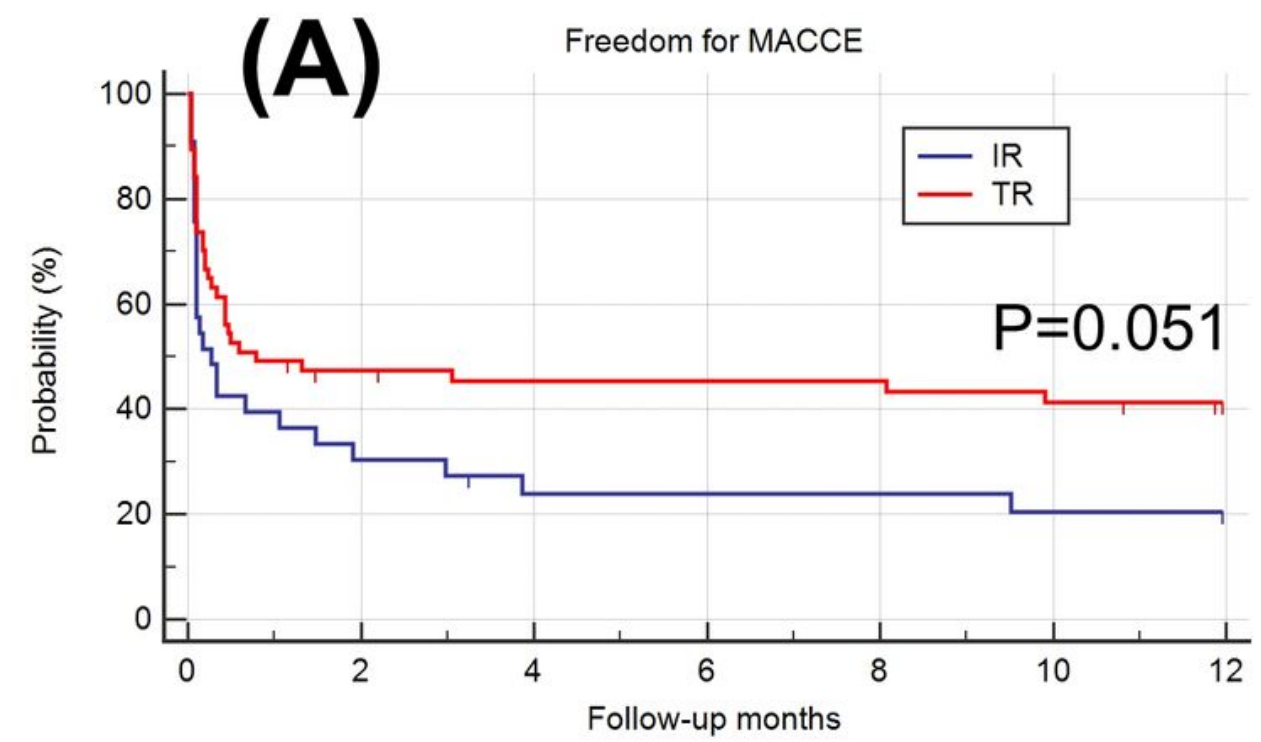

$\begin{array}{cccccccc}\text { IR } & 32 & 10 & 7 & 7 & 7 & 6 & 0 \\ \text { TR } & 32 & 25 & 23 & 23 & 23 & 21 & 0\end{array}$

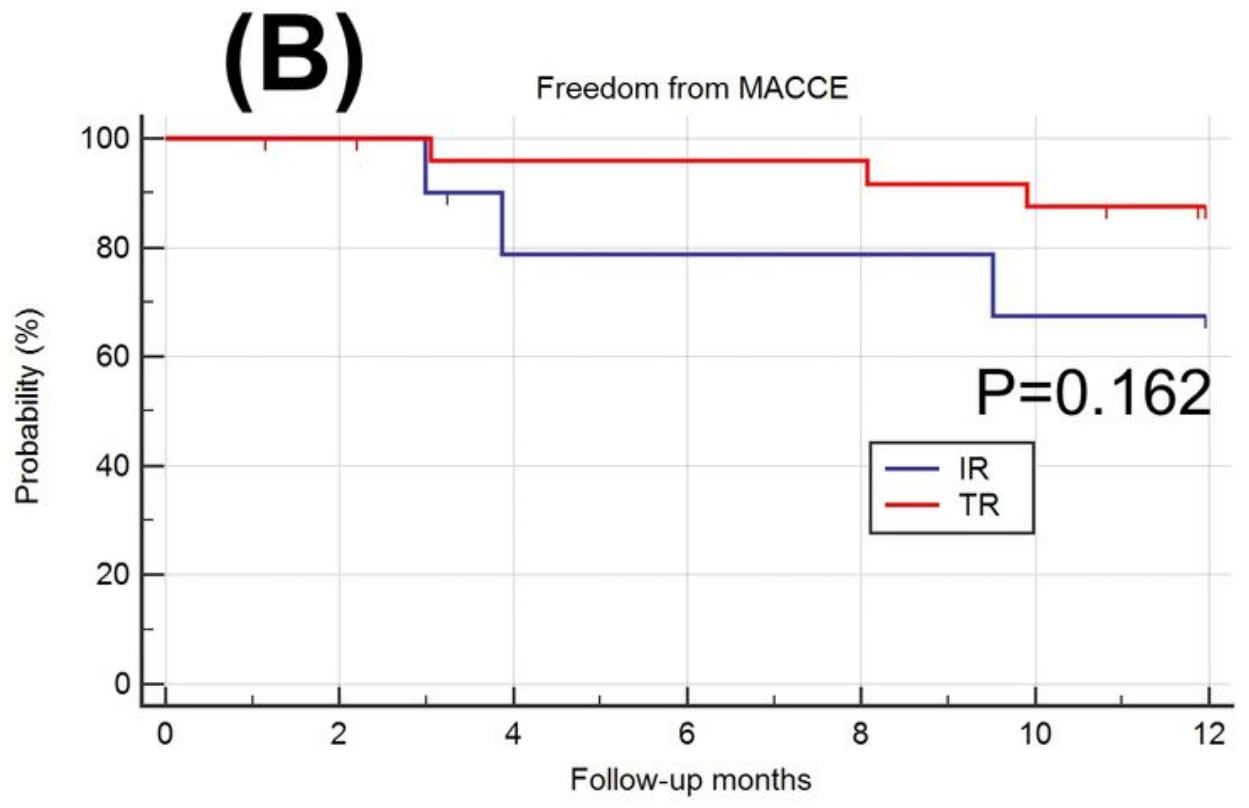

$\begin{array}{cccccccc}\text { IR } & 9 & 9 & 7 & 7 & 7 & 6 & 0 \\ \text { TR } & 28 & 25 & 23 & 23 & 23 & 21 & 0\end{array}$

Figure 2

Kaplan-Meier survival analysis for 1-year freedom from MACCE probability for all study patients $(A)$ and for hospital survivors (B). CR: complete revascularization, IR: incomplete revascularization. MACCE is defined by a composite eventsof cardiac death, myocardial infarction, revascularization, or stroke.

\section{Supplementary Files}


This is a list of supplementary files associated with this preprint. Click to download.

- SupplementTable1.docx 